Anuario del Instituto de Historia Argentina, vol. 17, n 2, e049, diciembre 2017.

ISSN 2314-257X

Universidad Nacional de La Plata.

Facultad de Humanidades y Ciencias de la Educación.

Centro de Historia Argentina y Americana

\title{
Los nuevos gobernantes de la Monarquía borbónica o el mundo de relaciones y senvicios de Bruno Mauricio de Zavala (1682-1736)
}

\section{The borbonic monarchy newest rulers or the world of relations and service of Bruno Mauricio de Zavala (1862-1736)}

\section{Rafael Guerrero Elecalde}

Universidad del País Vasco; Universidad de Córdoba, España | rafaelguerreroelecalde@yahoo.es

\section{PALABRAS CLAVE RESUMEN}

Elites El reinado de Felipe V supuso un momento de importantes cambios en la Monarquía hispánica. La

Redes Sociales aplicación de las primeras reformas también conllevó el cambio de las elites gobernantes, donde los

Felipe V.

Borbones colectivos periféricos fueron privilegiados por su especial lealtad a la causa borbónica durante la Guerra de Sucesión. Entre ellos, vascos y navarros fueron especialmente privilegiados, alcanzando las más cotas de

Buenos Aires

Corte poder, Así le sucedió a Bruno Mauricio de Zavala, que alcanzó los más altos cargos en la jerarquía militar y estuvo al frente de la gobernación de Buenos Aires. Familias, negocios, capitales, patrocinios, vínculos,

Poder servidumbres, contactos en la corte o servicio al rey fueron elementos fundamentales para conocer las formas de gobierno de la Monarquía de principios del siglo XVIII, lo que ayudará a desentrañar los fundamentos de la carrera del fundador de la ciudad de Montevideo como uno de los hombres principales

Militares de Felipe V.

\section{KEYWORDS}

Elites

Social Networks

Felipe V.

Bourbon

Buenos Aires

Court

Power

Militares

\section{ABSTRACT}

The reign of Felipe V was a period of important changes in the Hispanic Monarchy. The application of the first reforms also entailed the change of the ruling elites, where the peripheral groups were privileged because of their special loyalty to the Bourbon cause during the Succession War. Basques and Navarrese were especially privileged, reaching the highest levels of power. This happened to Bruno Mauricio de Zavala, who reached the highest positions in the military hierarchy and was in charge of the governorship of Buenos Aires. Families, businesses, capitals, patronage, bonds, easements, contacts in the court or service to the king were fundamental issues to know the forms of government of the monarchy in the beginning of century XVIII, which helps to unravel the foundations of the career of Montevideo's founder like one of the most important men of Philippe V.

Recibido: 10 de agosto de 2017 I Aceptado: 25 de octubre de 2017 I Publicado: 15 de diciembre de 2017

Cita sugerida: Guerrero Elecalde, R. (2017). Los nuevos gobernantes de la Monarquía borbónica o el mundo de relaciones y servicios de Bruno Mauricio de Zavala (1682-1736). Anuario del Instituto de Historia Argentina, 17(2), e049. https://doi.org/10.24215/2314257Xe049 
He recibido dos cartas del rey en que me honra con sus expresiones de su real benignidad, más propias de ella que de mis cortos méritos $\mathrm{y}$, aunque esto no vale oro para mí, es el tesoro más apreciable” 1 .

Así se expresaba en 1723 el vizcaíno Bruno Mauricio de Zavala (natural de Durango, 1682), gobernador de Buenos Aires, a su sobrino Martín Aurelio de Maguna y Zavala en una carta familiar. Este militar es uno de tantos vascos y navarros que con la llegada de los Borbones al trono español se participaron en la defensa de su causa durante la Guerra de Sucesión, en la aplicación de las primeras reformas borbónicas del siglo XVIII, así como en el ejercicio de su reinado en los vastos territorios hispánicos, llegando a los cargos más importantes en las distintas estancias de poder de la Monarquía. Negocios, servicio al rey, acción política y relaciones privilegiadas fueron siempre unidos y lo mismo sucedió con los diversos territorios y reinos; corte, Indias, provincias y comunidad local también estuvieron íntimamente vinculadas por la acción de estas familias.

En estas páginas no se encontrará una biografía del que fuera gobernador de Buenos Aires, sino un análisis de las tramas de relaciones en las que participó, las cuales estuvieron compuestas por parientes, amigos, patronos y otros agregados, principalmente naturales de las provincias del norte peninsular, que fueron fieles a la causa de Felipe V. Entre ellos se encuentran los nuevos gobernantes de la Monarquía.

\section{Hacia nueva monarquía y los hombres de Felipe V²}

A su llegada a España como rey, Felipe V se encontró con un escenario bien desfavorable. Era joven, inexperto, no había sido educado para ser rey, en unos territorios completamente ajenos (Albareda, 2010; Kamen, 2000; Martínez Shaw y Alonso Mola, 2001). Asimismo, llegó a una Monarquía sumida en una profunda crisis hacendística y de gobierno, donde las dotes de mando del soberano habían sido mermadas por las familias principales de reino gracias a la inoperancia de los últimos soberanos. Asimismo, el comienzo de la Guerra de Sucesión no hizo más que acrecentar dicha crisis (Kamen, 1974; Fernández Albaladejo, 2002).

Luis XIV puso especial interés por dirigir las políticas de gobierno que comenzaba a desarrollar su nieto. Por este motivo, envió a fieles agentes a la corte de Madrid para que le asesoraran. Principalmente, hizo introducir al embajador francés en el Consejo de Gabinete o de Despacho (Dedieu, 2000 y Castro, 2004) y a la princesa de los Ursinos en las casas reales (como camarera de la reina), por lo que esta dama se erigió como la principal baza dentro de los espacios menos “formales” de poder. De hecho, se convirtió en pieza fundamental, que se rodeó de hechuras que colaboraron con ella entusiastamente. Entre todas, la más importante fue Juan Orry, que llegó a España el junio de 1702 con el fin de subsanar las dificultades de la Hacienda real española. Éste fue el principal ideólogo del programa de reformas que se comenzó a aplicar en la Monarquía española, en colaboración de los miembros del partido profrancés que había sido clave para la implantación de la dinastía borbónica en España, con el específico objetivo del reforzamiento del poder del nuevo soberano a través de transformaciones en el aparato administrativo, hacendístico y 
militar (Dubet, 2008; Dubet, 2005; Badorrey, 1999; Desos, 2009, Dedieu, 2000).

Estas reformas administrativas se acompañaron por la renovación de los gobernantes de la Monarquía. En general, la alta nobleza castellana se vio desplazada de los espacios de decisión a favor del ascenso de otras familias, esencialmente de foráneos (franceses, italianos, irlandeses, flamencos), de la minoría aragonesa leal a la causa felipista y de las provenientes de la periferia de la Península (los llamados “norteños”). Este “colectivo” estuvo compuesto por un conjunto de familias bien relacionadas entre sí, que a lo largo de varias generaciones habían desarrollado una dinámica similar de negocios y carreras al servicio al rey, entroncando por vía matrimonial para establecer un “mapa familiar” que se extendió por los actuales territorios de Soria, Guipúzcoa, Vizcaya, Navarra, Álava, La Rioja, el norte de Burgos y la parte oriental de Cantabria. A lo largo del reinado de los Austrias consiguieron cargos de importancia en la alta administración, en el Ejército, en la alta jerarquía eclesiástica, en las secretarías o en los Consejos, perdurando en estas importantes posiciones hasta el inicio del reinado del primer Borbón.

Junto a estas familias participaron activamente a favor de la causa del duque de Anjou otras que, aunque también venían manejándose en el amplio marco de la Monarquía (principalmente con bases en el comercio colonial y las carreras militares en Flandes o Italia), no habían conseguido establecer consolidadas y duraderas bases en los espacios cortesanos. Desde entonces, y asociadas a las anteriores, consiguieron elevar a sus miembros a las más altas cotas de poder, suponiendo un espectacular espaldarazo en las posiciones de la casa y familia al formar parte del entramado del gobierno de la Corona.

Desde entonces, su principal activo fue un trato cotidiano con el monarca, que conllevó un mejor acceso a la gracia real y a los recursos de la Corona. Su acción modeló y definió, en toda su extensión, el tejido institucional, que sirvió también para la articulación, conformación y significado de los reinos y estados que la compusieron a ambos lados del Atlántico. De hecho, el fortalecimiento de la autoridad del soberano estuvo íntimamente relacionado con la configuración de vínculos que le ligaran con las elites de cada uno de los territorios, y la administración regia fue un vehículo predilecto para el establecimiento de dichas relaciones, ya que les permitía a estos grupos privilegiados salvaguardar o confirmar sus posiciones y poder (Guerrero Elecalde, 2012).

La casa fue la constructora y motor de estas dinámicas, en lo que correspondió a un aspecto más de la economía doméstica (la oeconomica) en la búsqueda de la conservación y aumento de su patrimonio material e inmaterial, por la que sus miembros fueron guiados por determinadas políticas pergeñadas en su seno y que fueron sustentadas por una obligada correspondencia entre todos ellos derivada de la participación de una “economía moral” que les comprometió a cumplir unos compromisos, adhesiones y contrapartidas, en la medida en que la ganancia personal estuvo inherente al buen funcionamiento de dicha economía y al triunfo colectivo dentro de una comunidad de intereses.

Estas estrategias también albergaron conflictos, alternancias y discontinuidades, y se aplicaron incluso a través de acciones contradictorias. Igualmente, estar bien relacionado, con amigos y parientes insertos en una dinámica exitosa repleta de cargos, influencias, capitales, honores, y en definitiva, poder, no implicó la consecución de éxitos y promociones. 
Una de las características principales de estas familias y casas fue el manejo y la acumulación de bienes patrimoniales y, especialmente, de cuantiosas cantidades de dinero procedentes (y en algunas ocasiones acumuladas desde hacía varias generaciones) del comercio y de negocios con la Corona y que pudieron reinvertir en diferentes operaciones durante la guerra y a favor de la causa de los Borbones. Desde bien temprano, la exportación desde los puertos del Cantábrico (como Bilbao, San Sebastián, Laredo o Santander) o por la ruta terrestre de Navarra de la lana llegada especialmente desde Burgos, hacia Francia, Flandes o Inglaterra.

Igualmente, los ferrones y comerciantes vascongados tuvieron en las Indias un excelente mercado. Sevilla se fue convirtiendo en el mercado más importante del hierro guipuzcoano y la primera parada antes de transportarlo a las plazas americanas ${ }^{3}$. Así, por ejemplo, en Buenos Aires, a principios del siglo XVII, el quintal de hierro valía veinticinco pesos, al llegar a Perú su precio se había incrementado en el 1.000\% (García Fuentes, 1991).

Los negocios se manejaron mejor desde un puesto en la administración y los comerciantes instalados en tierras americana solicitaron al rey (muchos desde el ámbito de la venalidad) empleos relacionados con sus inversiones: corregimientos, principalmente. En este sentido, los contactos en la corte fueron fundamentales para la consecución de empleos al servicio al rey, incluidos los que se otorgaron a través de una operación venal. Los oficios conseguidos en América sólo pudieron responder a poderosas conexiones en la corte y en la alta administración de la Monarquía (compuestas por parientes, amigos y otros agentes); un complicado proceso que requería la presentación de memoriales, conversaciones con los nombres influyentes, acceso al rey y la eliminación de competidores.

Estas actividades mercantiles supusieron un impresionante manejo y acumulación de capitales que repercutieron directamente en la casa nativa y, por extensión, en la comunidad de origen. Los parientes colocados poderosamente mandaron habitualmente remesas y ayudas a los familiares (las más conocidas en el momento de su muerte) que se invirtieron tanto en las carreras de los jóvenes en el servicio de "ambas majestades", así como de la colocación de las hijas en matrimonios ventajosos y en conventos, como en la ampliación y mejora del patrimonio familiar, a través de la compra de tierras, negocios u otras dependencias (Imízcoz, 2001).

Bruno Mauricio de Zavala no fue ajeno a este mundo. Griselda Tarragó explica cómo su padre, Nicolás Ibáñez de Zavala y Churruca (Durango, 1629), realizó su carrera en el virreinato del Perú (salió de casa con trece años) en estrecha relación con sus primos, los Bengolea, naturales de Guizaburuaga. Diversificó sus inversiones y actividades económicas en variados frentes, y obtuvo cuantiosas ganancias (entre otros: comercializó cobre con Lima y Santiago de Guatemala; traficó con géneros y mercaderías de navíos llegados de la flota de Indias, tanto en la Nueva España como en la Tierra Firme; prestó dinero a diversas personas de Madrid y del comercio común de Sevilla, entre ellos se contó, por ejemplo, el guipuzcoano Antonio de Isasi, que lo utilizó para la adquisición de la gobernación y capitanía general de Chile en una operación venal; realizó otros empréstitos de plata y escrituras de obligación a vecinos de la Villa de Madrid y el comercio común de la ciudad de Sevilla). Nicolás Ibáñez del Zavala dirigió siempre una parte de las ganancias económicas a adquirir bienes raíces en el país que sirvieron para ampliar la hacienda de su casa nativa, en Durango, y para elevarla aún más decidió someter sus bienes en un mayorazgo en 1677. También aportó dinero para 
la carrera y educación de sus hijos naturales, ubicados como militares en Flandes, vecinos de Lima, quienes desarrollaron la carrera en Indias o en la Universidad de Salamanca (Tarragó, 2017b).

Tal como señala Francisco Andújar, en muchas ocasiones, el “sonido del dinero” tintinea detrás de la obtención de honores y cargos al servicio del rey, quien habilitó en estos primeros años de reinado la compra de los mismos a través de la Secretaría del Despacho y de su financiero Juan de Goyeneche. (Andújar, 2004).

En definitiva, estas familias vascongadas (y “norteñas”, en general) se implicaron íntimamente con los miembros del equipo de gobierno enviado por el rey de Francia y en los valores y espíritu que representaba. Estos agentes (también las familias del colectivo norteño) fueron los encargados de dar contenido a las reformas borbónicas, otorgando el sentido deseado, así como haciendo posible su ejecución y práctica. Dentro de las posibilidades, los elegidos para estos empleos fueron hombres pertenecientes o muy cercanos al equipo de gobierno de Felipe V que encabezaba la princesa de los Ursinos por lo que los caminos trazados por los vínculos y afinidades, reconfiguradas en cada acción, fueron las vías más seguras para las órdenes reales.

Sin embargo, este proceso no siguió un camino lineal. En los primeros años de reinado de Felipe V se constituyeron alrededor de su figura distintas camarillas que protagonizaron importantes luchas por el control de sus decisiones, lo que llevó incluso a la caída en desgracia de algunas de sus cabezas y, por ende, de sus más fieles adeptos. Así pasó con el destierro de la princesa de los Ursinos durante unos meses en los años 1704-1705, que conllevó tanto la paralización de las reformas como a la caída de todos sus adeptos. Con su vuelta, se restableció la situación anterior.

Las disposiciones originadas en las altas esferas de poder de la Monarquía se trasmitieron siguiendo el orden jerárquico administrativo establecido, que a su vez estuvo conformado por amigos, parientes o paisanos adeptos a la causa y que añadieron a su correspondencia “oficial” expresiones de afecto y lealtad. Por lo tanto, no existió una frontera (o fue casi imperceptible) entre la administración y las relaciones personales, por lo que más allá de un compromiso individual, las medidas respondieron a la movilización coordinada de la red de parientes y allegados para la ejecución de las mismas. (Guerrero Elecalde, 2012).

Tras el fin de la Guerra de Sucesión, y la consiguiente consolidación de Felipe V en el trono, se produjo también el afianzamiento de las familias que apoyaron incondicionalmente su causa durante el conflicto bélico. El compromiso de fidelidad demostrado a lo largo de esos años hizo posible que obtuvieran la plena confianza del soberano, lo que les permitió alcanzar los espacios más importantes del poder de la Monarquía. Fue tal su elevación en la corte que sus detractores vieron en ellos un grupo de presión al que denominaron "partido vizcaíno", aunque como hemos podido comprobar, más que una facción de carácter político fue una asociación de familias originarias de las provincias vascas y de otras, coaligadas a las anteriores por compartir, por lo menos en algún momento, negocios, inversiones e ideario comunes. Sus momentos más álgidos comenzaron en la década de 1720, tras unos años de incertidumbre política tras el fin del conflicto bélico. Algo inaudito hasta entonces en lo que respecta a su poder y a la envergadura del fenómeno.

Desde la corte, centro neurálgico del poder, estas familias extendieron sus redes, por medio del acceso a nombramientos en cargos al servicio al rey, a otros espacios y a territorios de la Monarquía 
(la corte, la provincia o Indias) (Benigno, 1994). La dinámica de estas familias excedió el marco compartimentado de la institución. Estos empleos albergaron cotas de poder, de decisión y de gobierno, en muchas ocasiones de especial importancia para el devenir político y fueron una herramienta muy valiosa utilizada por estas redes en beneficio de sus políticas familiares, lo cual mejoró notablemente la economía de la casa y favoreció la colocación de sus vástagos en diversos destinos vinculados a la Monarquía, que ayudarían a la consecución de recursos de carácter económico y honorífico. Estos recursos consolidaban la posición de linaje y de la casa en su comunidad, lo cual era necesario dado que el reclutamiento de los servidores del rey se hacía desde la lógica de la casa y de la geometría de las relaciones.

Paralelamente, estos personajes instalados en la corte consolidaron sus bases en sus comunidades de origen y en las provincias (lo que era parte de su poder en la corte). Esto fue gracias a la labor de familiares, de hombres de confianza o de medio parientes instalados en las villas o aldeas nativas, los cuales informaban de todo lo acontecido en las reuniones de las juntas o de los concejos y presentaban en ellas los dictámenes de estos preclaros hijos de la tierra, para poder así influir en las políticas de estas corporaciones. Ellos también fueron los que que capitalizaban los triunfos y el ascendiente de esos prohombres en la comunidad. Desde otra perspectiva, las diputaciones encontraron en estos hijos de la Provincia o Señorío a los principales protectores de sus intereses en la corte, por lo que fueron nombrados en muchas ocasiones como sus delegados ante el rey para la resolución de diferentes dependencias o conflictos, aunque también pudieron defender la causa de la Provincia ante las autoridades reales sin un nombramiento previo (Guerrero Elecalde, 2017).

Siguiendo el caso de Bruno Mauricio de Zavala, dentro de esta política reformista se inició la remodelación del ejército (ineficaz ante los nuevos tiempos) con el objetivo de la formación de unos cuerpos modernos y permanentes dedicados tanto a la defensa exterior como a la labor de policía en el interior.

En el momento en el que se produjo un verdadero aumento del poder del soberano fue tras las reformas de las tropas de la casa real, para dejar de ser meras tropas palaciegas (especialmente dedicadas al ceremonial) y convertirse en cuerpos de elite y tropas de primera línea en el combate. De este modo, el 22 de septiembre de 1702, Orry fundó el regimiento de guardias de caballería del rey, y eligió como coronel al vasco de Vitoria Francisco Antonio Agurto y Salcedo, marqués de Gaztañaga y antiguo gobernador de Flandes, sin embargo, éste falleció precipitadamente el 2 de noviembre de ese mismo año. La construcción de estos cuadros del ejército causó gran controversia entre los gobernantes. Para algunos, estas medidas fueron muy novedosas que, además, que les otorgaban un importante poder político, por lo que Orry, en estos primeros años de reforma militar, decidió contentar al cardenal Portocarrero, al duque de Veragua y a Ronquillo nombrándoles sucesivamente coronelel de dichas guardias.

En noviembre de 1703 se instauró un cambio radical con la constitución de cuatro compañías de guardias corps (dos españolas, una italiana y una valona) y dos regimientos de guardias de infantería (uno de guardias españolas y uno de guardias flamencas). Aquellos que desempeñaron empleos en dichos cuerpos, además de asegurar rápidos ascensos en el escalafón militar, alcanzaron el generalato e importantes mercedes de carácter honorífico, como hábitos, encomiendas y títulos nobiliarios y guardaron un especial prestigio por la relación con el rey. Igualmente tuvieron la 
posibilidad de conseguir gobiernos político-militares, ya fueran virreinatos, capitanías generales o gobernaciones, y tuvieron un papel fundamental en la Corona de Aragón -reinos desafectos a Felipe $\mathrm{V}$ durante la guerra y que merecieron una especial atención tras su derrota, y en las Indias, donde también ejercieron una labor esencial para el establecimiento de las políticas reformadoras en estos reinos (Andújar, 2004 y 2000).

Esta posición privilegiada de los guardias reales se contempla en una carta personal de Bruno Mauricio de Zavala, a punto de trasladarse a Buenos Aires para ejercer de gobernador": "ya sabrás como el rey me hizo mariscal de campo de sus ejércitos. Me ha prevenido el secretario del Despacho que me reserve esta noticia hasta el embarco por el ejemplar y así tú no las publiques, aunque te digan todos que la saben"

Es de destacar en lo que respecta a América, y desde una perspectiva en clave familiar, cómo muchos de estos guardias fueron dirigidos a destinos que no les eran del todo desconocidos, ya que sus casas y parentelas venían comerciando desde hacía varias generaciones con productos europeos (especialmente hierro traído desde las provincias vascas, muchas veces elaborado en sus propias ferrerías) y también venían trabajando por la captación de bienes indianos (Guerrero Elecalde; Tarragó, 2014), como la plata del cerro del Potosí o de las minas de Zacatecas, la yerba mate santafesina o el cacao venezolano.

Este aspecto también lo confirma el caso de Bruno Mauricio de Zavala y su parentela, si lo contemplamos con la creciente consideración del puerto de la ciudad de Buenos Aires y el camino interior que unía Lima y Potosí (Tarragó, 2017b). También podemos observar estas prácticas en Venezuela, donde operaba la Real Compañía Guipuzcoana de Caracas desde 1728. Para este destino fueron elegidos los guipuzcoanos Martín de Lardizábal y Elorza, comandante de la Provincia de Venezuela desde 1732 y Gabriel José de Zuloaga y Moyúa, su gobernador desde 1736 (Guerrero Elecalde, 2012).

Por una parte, estos gobernadores y virreyes, fueron los garantes de los intereses de Felipe $\mathrm{V}$ en estos territorios $\mathrm{y}$, en su nombre, actuaron buscando posiciones favorables en un contexto desfavorable debido a la acción e influencia de otras potencias europeas. Sin embargo, se debe tener en cuenta que la acción de estos agentes se articuló en una multiplicidad de ámbitos simultáneos y en una configuración compleja, cuya forma y dinámica se vinculó desde los objetivos del rey, pasando por intereses particulares y la lógica de la fidelidad personal (Moutoukias, 2002).

\section{Juan de Idiáquez, hombre principal en las guardias y en el palacio}

Perteneciente una importante familia de Guipúzcoa, con gran prestigio y poder desde el siglo XVI무, Juan de Idiáquez (nacido en Azcoitia, 1665) destacó desde los primeros años de reinado de Felipe V. Cuando contaba con la edad de dieciséis años comenzó sus servicios en el ejército con el grado de capitán, y desarrolló una importante carrera en Flandes, donde fue maestre de campo de un tercio viejo de españoles (Andújar, 2009). Su buena relación con el marqués de Gaztañaga, primer coronel de las guardias reales (y medio pariente), le llevó a la corte para diversas cuestiones, en especial las relacionadas con la reforma militar, por lo que se implicó de este modo en las medidas adoptadas por la facción encabezada por la princesa de los Ursinos. Tras las primeras reformas en las tropas de 
las casas reales, en 1704 fue nombrado sargento mayor de las guardias de corps. Entre sus funciones, se encargaba del nombramiento o la consulta al rey de los empleos administrativos y técnicos del estado mayor de la guardia, por encima de las compañías.

La elección de los miembros de estos primeros regimientos (pero también de los sucesores) se realizó de forma grupal, siendo determinante para el reclutamiento el factor familiar. Con el tiempo, en muchas ocasiones los empleos fueron ocupados sucesivamente por miembros de la misma parentela o de las familias amigas.

De este modo, solamente como consecuencia de estas prácticas y de la influencia fundamental Juan de Idiáquez, se puede explicar la importante presencia de vascongados en estas primeras promociones. Desde su privilegiada posición, comunicó puntualmente a sus familiares de los dictámenes resueltos en la corte, y construyó con esta "información privilegiada”, y junto con los demás miembros de su familia, las políticas más favorables para la parentela. En enero de 1704, el sargento mayor de las guardias de corps escribió a su hermano Pedro, dueño de la casa familiar, para informarle las condiciones impuestas para el reclutamiento de cadetes de las guardias reales, el número de componente por cada compañía así como sus sueldos, y resaltó las grandes posibilidades que se ofrecían. Igualmente, le brindó la oportunidad para que desde Azcoitia le seleccionase y presentase a los miembros de la parentela y de otras de familias amigas más adecuados para entrar a formar parte de estas guardias ${ }^{7}$.

Entre los principales protegidos de Juan de Idiáquez, que desarrollaron su carrera en las guardias reales estuvieron, entre otros, Miguel Antonio de Zuaznábar, que comenzó su servicio en las guardias de corps en 1707 y ejerció como cadete desde el primero de mayo de 1711 y en todos sus nombramientos aparece la firma de su mentor ${ }^{8}$. La buena relación que los miembros de la familia Idiáquez mantenían con los Zuloaga, de Fuenterrabía, a su vez parientes de los Olazábal, fundamentó también en un lejano vínculo y se extendió más allá de Guipúzcoa. Idiáquez compartió amistad con Juan Bautista, que debió pasar largas temporadas en la corte, aunque fue canónigo maestrescuela de la catedral de Cádiz y trabajó por los ascensos de su hermano Gabriel José de Zuloaga y Moyúa (natural de Fuenterrabía, 1672) en las guardias reales españolas.

Asimismo, Joaquín de Emparan y Azcue llegó a ser primer teniente de granaderos del regimiento de guardias españolas, y encontró su muerte en diciembre de 1710, defendiendo la causa felipista en la

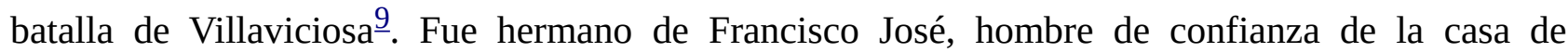
Idiáquez y de José Grimaldo, secretario del Despacho de Guerra y Hacienda.También entre estas familias se encontraban los Zaldúa, que introdujeron a los hermanos José Ignacio y Antonio Miguel de Zaldúa y Gamboa en las primeras promociones de las guardias de corps. Naturales de Bilbao, desde jóvenes fueron destinados al servicio de las armas y no hubo mejor destino que desarrollar su carrera bajo el amparo de Juan de Idiáquez $\underline{10}$.

Otro de los protegidos principales de Juan de Idiáquez fue Bruno Mauricio de Zavala, que debió su entrada en el cuerpo militar a su amparo. Cuando era joven fue aviado a Flandes para comenzar su carrera en los tercios y como reza en su expediente para el ingreso en la orden de Calatrava en 1701, fue “alférez coronel del tercio de D. Juan de Idiáquez en el ejército de Flandes” 11 . Desde entonces, fue ascendiendo en el escalafón para alcanzar de este modo el grado de teniente de su compañía. 
Bruno Mauricio siguió ligado a la figura del cada vez más poderoso Idiáquez, quien siguió influyendo en su carrera. A los veintidós años ya tenía el grado de capitán y se le concedió la merced del mando de una compañía en el tercio.

Tras la constitución de las guardias reales y de corps, en 1704, lo que coincidió también con el nombramiento de Juan de Idiáquez de sargento mayo de las guardias de corps, regresó a España y entonces fue elegido primer teniente del regimiento de infantería española. Comenzó a servir desde el 1 de febrero del año siguiente. En 1709 fue elevado a capitán $\underline{12}$. Sus actuaciones para la constitución del regimiento de Vizcaya de 1709 fueron premiados con un ascenso a brigadier de infantería de los reales ejércitos $\underline{13}$.

El poder de Juan de Idiáquez se vio aumentado sobremanera cuando consiguió la confianza de los monarcas y fue elegido para el gobierno y la educación de la casa y persona del infante Don Fernando. De este modo, en 1721, fue nombrado como gobernador del nuevo cuarto separado en palacio del infante don Fernando, con retención de su empleo de sargento mayor de guardias de corps, $\underline{14}$ y en 1724, ayo del Príncipe de Asturias -por el fallecimiento de su hermano Luis I -, el futuro Fernando VI. En 1725, sumiller de corps del Príncipe de Asturias. $\underline{15}$

Desde este puesto en palacio tuvo una relación personal con los reyes e infantes, por lo que dispuso de un acceso directo a la gracia real. Uno de sus patrocinados confirmó que era "el segundo espejo de España”, así en su justo proceder como de política y el príncipe le tiene en lugar de su padre totalmente con una obediencia y agradecimiento inexplicable”. $\underline{16}$

Juan de Idiáquez también se elevó poderosamente en la escala de honores de la Monarquía. Obtuvo

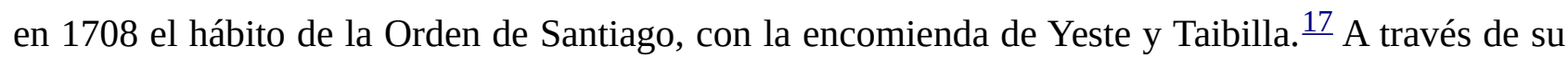
matrimonio con su sobrina Mariana Velasco e Ibáñez de Segovia, disfrutó de los títulos de conde de Salazar y de conde de Castilnovo. Posteriormente, y en recompensa por los servicios prestados a la Corona, se le otorgó el Ducado de Granada de Ega con la grandeza de España de primera clase. $\underline{18}$ Murió el 9 de septiembre de 1736

\section{Pedro Bernardo Villarreal de Bérriz, un "cortesano de provincias"}

En el Antiguo Régimen, la Monarquía estuvo compuesta por la agregación de sus territorios que se integraron a través de diferentes adscripciones (por conquista, por agregación patrimonial y por herencia dinástica), por lo que el soberano se convirtió prácticamente en el único vínculo de unión articulador entre los dominios y árbitro garante de las normas y leyes de cada reino y corporación (Mazín, 1998; Yun, 2009; Barriera, 2002 y 2002b; Esteban, Ruiz Ibáñez, 1998). Más allá de un sistema centro-periferia, se trata de un mundo dinámico, en el que los límites de cada una de esas instancias se halla en un proceso de redefinición constante (Gil, 2013) y, aunque la corte fue el centro neurálgico del poder y el lugar en donde el monarca dispensó su gracia, la geografía de los vínculos construida por estos grupos familiares se caracterizó por una geometría variable (Gil, 2017; Arrieta, 2009-2010; Arrieta, Gil, Morales, 2017; Clavero, 1991; Hespanha,1993).

Para estas familias no hubo una diferenciación de espacios (el americano, el cortesano, el provincial), sino que, a través de sus tramas y vinculaciones, promovieron las movilizaciones de sus 
miembros por unos caminos con fronteras imprecisas e ilimitadas y que no contemplaron las dificultades que podían imponer las grandes distancias de la Monarquía (Guerrero Elecalde, 2012).

Pedro Bernardo de Villarreal de Bérriz y Andicano (nació en Mondragón, 1669) vino al mundo en el seno de una familia muy conectada con los diferentes espacios de poder de la Monarquía y desde sus primeros años fue criado en la corte. Y es que su madre, María Sáez de Andicano Celaá (natural de Mondragón, 1627) $\underline{19}$, fue azafata de la infanta Margarita Teresa de Austria, prometida del futuro emperador Leopoldo I;además Juan de Andicano, el tío de María, fue el primer conde de Monterrón, y llegó a ser en 1680 consejero de Castilla $\underline{20}$. Sus padres estaban casados en segundas nupcias $\underline{21}$ y ambos contaban con descendencia de sus uniones anteriores por lo que, aunque no tuvo hermanos enteros, contó con un buen número de medios hermanos con los que conservó una estrecha relación a lo largo de su vida.

Entre ellos destacó su hermano de padre, Juan Bautista Villareal (natural de Gámiz, 1655)ํㅡㄹ que fue fiel servidor de Luis Francisco de la Cerda, duque de Medinaceli, a quien sirvió desde su etapa como marqués de Cogolludo en Flandes. También lo hizo como su camarero mayor cuando éste, en 1695, fue nombrado virrey y capitán general de Nápoles.

El 3 de enero de 1694, Pedro Bernardo contrajo matrimonio en Lequeitio con Mariana Rosa de Bengolea, principal heredera de su casa familiar. Desde entonces, pasó a residir en la torre de Uriarte, sita en dicha villa vizcaína, y se colocó a la cabeza de los bienes que heredó de su padre, así como de las casas, solares y resto del patrimonio, material e inmaterial, de su familia política, los Bengolea. Esta familia, con su casa ubicada en Guizaburuaga, se dedicaba, desde por lo menos la segunda mitad del siglo XVI, a la producción y posterior exportación del hierro elaborado en sus ferrerías a diferentes plazas comerciales del norte de Europa y a las Indias $\underline{23}$.

Del mismo modo que su familia política, Pedro Bernardo continuó con estas producciones y exportaciones a la gobernación de Buenos Aires y al virreinato del Perú, lo que le permitió invertir los importantes caudales llegados desde Indias en la mejora o la ampliación de sus propiedades vizcaínas. Según sus propias palabras: "Desde que me emancipé a los diez y ocho años de mi edad (en que emprendí el reedificio desde los cimientos de unas herrerías) en cuarenta y ocho años continuos apenas me han faltado obras en mi hacienda y la de mis hijos siendo las más en herrerías y molinos” (Larrañaga, 1974).

Los Villarreal de Bérriz, Andicano y Bengolea fueron familias muy reconocidas tanto en el Señorío como en la provincia de Guipúzcoa y, por lo tanto, Pedro Bernardo fue y será un personaje altamente considerado y valorado, no sólo por la calidad de su linaje y el origen de su hidalguía, sino también por su capacidad personal y sus buenas acciones, fundamentadas en su condición de hombre honorable y buen jefe de familia. Pedro Bernardo, vecino y residente en la villa vizcaína de Lequeitio, contó desde principios del siglo XVIII con familiares y allegados en el Consejo de Castilla, en la Secretaría del Despacho Universal, en los altos cuadros militares de las guardias reales y de corps, en la alta jerarquía eclesiástica,, entre otras instituciones, por lo que tenía la oportunidad de llamar a la puerta de las más altas instancias.

Asimismo, contó con parientes en distintos puntos de América, como los Esquivel, los poderosos y 
muy ricos mineros del Cuzco, también asentados en Lima, o los propio Bengolea, comerciantes de hierro con las Indias. Entre ellos cabe destacar la relación con los Ibáñez de Zavala, más concretamente entre Pedro Bernardo Villarreal de Bérriz y Bruno Mauricio de Zavala, que se afianzaron con el matrimonio del primero.

Su capacidad personal, así como sus importantes relaciones, convirtieron a Villarreal de Bérriz en un personaje clave para el buen desarrollo de las políticas familiares, tanto en las Indias como en la Península, lo que le permitió situarse como el patrón principal en esta parte de Atlántico con el manejo de diferentes cuestiones de vital importancia para la parentela y también para los aliados y allegados. Fue uno de los encargados de conseguir el hierro necesario para comerciar y, probablemente también, de obtener otras mercaderías para enviarlas a Sevilla, como puente hacia América. Una parte del hierro se elaboró en las factorías de la familia, con el combustible que le procuraban las propias explotaciones forestales, y quizás también con una flota de fabricación propia, construida asimismo de la madera de sus montes y de los productos de sus ferrerías $\underline{\underline{24}}$.

Al mismo tiempo, desde la torre de Uriarte, Pedro Bernardo movilizó a gente de su confianza (especialmente familiares y amigos) para situarlos en los espacios y lugares adecuados para sacar adelante diversas operaciones beneficiosas para la casa, seleccionando a los agentes más convenientes en cada lugar, preparados y alertas para actuar ante el nacimiento de cualquier oportunidad. Estos actores operaron como fieles aliados, pero también como socios o encargados de negocios eventuales, conformando unas tramas relacionales que de forma tentacular pudieron llegar a diferentes recursos y espacios de poder de Indias y de las Península.

De este modo, servicios, favores y recursos fluyeron en múltiples sentidos por la compleja trama de la Monarquía, en la que las largas distancias se estrecharon gracias al continuo intercambio de noticias a través de cartas, repletas de las interpelaciones e invocaciones a la fidelidad, correspondencia, la lealtad y el honor, fundamentos primordiales en el imaginario y devenir de estos actores y en las lógicas de estas tramas relacionales $\underline{25}$.

Como los asuntos no fueron lineales, estos personajes tuvieron que diseñar las estrategias más convenientes y políticas de acuerdo al devenir de los acontecimientos. La acción de Pedro Bernardo Villareal de Bérriz muestra, dentro de este contexto general de relación entre las necesidades de la Monarquía y los negocios particulares, cómo los hombres de las provincias relacionadas especialmente con la corte podían aprovechar esta relación para reorientar sus negocios.

En un contexto de crisis de la industria ferrona, la Guerra de Sucesión conllevó el cierre de las exportaciones del hierro vizcaíno por la paralización del tráfico a Indias y la pérdida de los territorios europeos. En esta situación, Pedro Bernardo se trasladó a Madrid con el objeto de solicitar un asiento para fabricar armas y pertrechos en sus ferrerías de Bengolea, algo inaudito hasta entonces. En 1705 cerró con Felipe V un asiento por seis a ocho años para "hacer en sus ferrerías de Bengolea, que son las mejores de todo aquel Señorío la fabricación de bombas, carcasas, balas, granadas y todos los demás pertrechos de guerra que se fabrican en las oficinas de Eugui, en Navarra y en las de Liérganes (...)” (Ruiz de Azúa, 1990).

Este asiento requería unas inversiones y suponía unos gastos. Había que adecuar las ferrerías, construir los ingenios de artillería y transportar la producción a los destinos ordenados por la 
Secretaría del Despacho de Guerra y Hacienda, en un espacio muy amplio entre la frontera de Portugal y los puertos del Mediterráneo. A cambio, Pedro Bernardo no sólo aseguraba la salida del hierro de sus ferrerías y ganaba dinero, sino que obtenía del rey el privilegio de "real fábrica" durante la duración del contrato, que le concedía todos los honores y franquicias que tenían las fábricas reales de esta calidad. Esto se hacía extensivo a su casa de Bengolea y a los administradores y oficiales que trabajaban en ella otorgándoles la calidad de "maestros artilleros de España”.

Una empresa de tanta envergadura fue posible gracias a la participación de diferentes parientes y allegados de Pedro Bernardo que colaboraron en los abastecimientos de las ferrerías, la elaboración de los pertrechos y el acarreo de la producción a los destinos. En este papel fue fundamental el concurso del pariente Nicolás de Ubilla y Munibe y de Pedro de Aguirre, que fueron copartícipes de las estrategias que se estaban concretando en la corte ${ }^{26}$.

Años después, Pedro Bernardo preparó la formación de sus hijos, Pedro José (cariñosamente, Peru) e Ignacio. Tras pasar por el Colegio de la Compañía de Jesús de Tolouse (1716-1719) les avió a Madrid para pasar un tiempo en la corte. Ignacio, como sucesor de las posesiones familiares, debía ejercitarse en algunas cosas de su edad y obligadas de su condición (montar a caballo, tomar lección de espada y de danza y frecuentar los círculos nobiliarios para ser conocido) y después regresara a Lequeitio para ayudarle con los asuntos domésticos y completar su preparación. Otro de sus cometidos fue buscar colocación y orientar la carrera de Peru. Ya tenía quince años y la inclinación del muchacho en la carrera de las armas era evidente, por lo que se afanó por introducirle en las guardias de infantería española.

Uno de los contactos de la familia en estos cuerpos fue el teniente coronel José de Armendáriz, navarro y futuro marqués de Castelfuerte, que había forjado amistad con el dicho Juan Bautista de Villarreal desde su etapa en el reino de Nápoles. Por este motivo decidieron explotar dicho vínculo y colocarlo como cadete en su compañía.

En este cuerpo militar, el máximo representante del cuerpo después del rey y su coronel de las guardias de infantería española era Guillén Ramón Moncada Portocarrero, marqués de Aytona, quien guardaba un vínculo lejano con los Villarreal de Bérriz. Su hermano, Manuel Pedro, estaba casado con Teresa María de la Cerda, IV condesa de Baños, que tenía ascendencia vizcaína $\frac{27}{\text { y }}$ guardaba relación de parentesco con la casa de Medinaceli, a quien los hijos del señor de Bérriz habían servido durante años. La participación de diferentes miembros de estas grandes familias a favor de la causa de Peru también debía ser fundamental para la consecución de los objetivos: debían convencer de la obligatoriedad de promocionar al joven vizcaíno al coronel de las guardias $\underline{28}$.

Al día siguiente de su llegada a la corte, decidieron no perder el tiempo y comenzaron a realizar las visitas oportunas para lograr cuanto antes la plaza a Peru, a la vez que hicieron llegar a Armendáriz una carta de su tío:

"Esta tarde hemos encontrado al tiempo de irle a ver a Armendáriz, a quien hemos entregado la de mi tío. Nos ha dicho le ha hablado de nosotros el duque de Medinaceli, y en fin, hemos quedado en irle a ver mañana, como al conde de Baños, asegurándonos 
todos que la mejor recomendación para el teniente coronel es la de mi tío, por la gran amistad que tiene como nos lo ha dicho la marquesa de Priego" $\underline{29}$.

Aunque los trámites eran complicado, gracias a los apoyos con los que contaba la familila, al poco tiempo de su llegada a Madrid, Peru sentó plaza de cadete de las guardias de infantería española en la compañía de José de Armendáriz $\underline{\underline{30}}$. Tras la consecución del asiento, la familia no paró y empezó a trabajar para el logro de una bandera para Peru; se trató de un destino y empleo dentro de dicha unidad que facilitarían sus ascensos en el escalafón de las guardias de infantería española. Este objetivo se presentaba especialmente complicado por la escasez de plazas vacantes (parece que había sólo tres) $)^{31}$.

Las bases de la familia eran de mucho peso, pero todo esfuerzo se hacía escaso para una tarea de tanta dificultad. Los esfuerzos de los hermanos Villarreal de Bérriz estuvieron relacionados con frecuentar las casas más importantes, y a los guardias más influyentes (entre otros, Blas de Loya, José de Armendáriz, el propio Aytona), así como a presenciar los actos relacionados con la familia real y poder entrar en palacio, participando como uno más de la corte. En estos aspectos tuvieron un importante apoyo en Juan de Idiáquez y Carlos de Areizaga y, en una menor medida, en la marquesa de Montehermoso.

Así, por ejemplo, los muchachos acudieron a El Retiro, donde los soberanos buscaban habitualmente esparcimiento junto a sus servidores más cercanos. Allí, tanto Carlos de Areizaga como su pariente y mentor Juan de Idiáquez, que por sus empleos acompañaban al Príncipe de Asturias y a los infantes, se paraban a hablar con Ignacio y Peru a la vista del resto de la corte, elevando así su calidad ante los observadores:

"Nosotros, a Dios gracias, buenos, logrando de los buenos tiempos que hace y mayormente con estar los reyes en El Retiro acudimos allá todas las tardes y entramos en el cuarto de Idiáquez y Areizaga, donde somos bien recibidos. (...) Ayer hablamos a mi señora la marquesa de Montehermoso [de la parentela de los Idiáquez y aya de los infantes], pregúntonos de v.m. y D. Francisco Antonio, su hijo, con muchos b.m. para v.m.,$\underline{32}$

Estas buenas relaciones entre los Idiáquez y los Villarreal de Bérriz, fundamentadas desde hacía tiempo, también fueron cultivadas por los chicos. Dentro del intercambio de servicios de ambas casas, se interesaron y colaboraron por sacar adelante negocios que los de Azcoitia tenían pendientes en la corte.

Además, en esta labor de dejarse ver y ser visto, ante la incipiente promoción los cadetes pretendientes a las banderas vacas rondaron en palacio en torno al marqués de Aytona para no ser olvidados por su superior; el joven Villarreal, por su parte, se presentó como uno más para no perder posibilidades y en este juego salió fuertemente recompensado:

“Todos los cadetes están creyendo fijamente que Peru se llevará una bandera, que es la razón porque el marqués de Aytona le habla alguna vez delante de los otros, mayormente en tocando pieza de Francia, y todos los oficiales le hablan con distinción de todos los demás cadetes, y rabian sus compañeros de verle con mucho más decente porte que ellos y admitido en los correos de oficiales" $\underline{3}$. 
La manutención de los jóvenes en la corte dependió exclusivamente de la casa. Además, desde Lequeitio, Pedro Bernardo les envió diversas viandas elaboradas en casa para asegurar que los chicos estuvieran alimentados correctamente y para que los repartiera entre parientes y otras obligaciones. Así ocurrió con la llegada de varios barriles de escabeche con carne, pimientos, besugo y congrio: "La repartición se ha hecho uno a Jarabeitia, dos a Armendáriz, y dos para casa" $\underline{34}$.

En estas relaciones -y también para conseguir una bandera en las guardias de infantería española o agradecer favores recibido-, valían tanto los méritos del muchacho, la casa a la que pertenecía y los importantes apoyos en la corte, como también agasajos y regalos (esta vez en forma de escabeche) destinados al teniente coronel Armendáriz, con quien, según parece, se reunían a menudo.

Desde su primer destino en la compañía del teniente coronel de guardias José de Armendáriz, en el cuartel de San Feliú (cerca de Barcelona), el 4 de octubre de 1721, Peru llevó a cabo una espléndida carrera militar, dictada también por la senda del parentesco y las buenas relaciones con sus superiores. Así se lo exponía Pedro Bernardo a Bruno Mauricio: "Peru sentó luego plaza de cadete de las guardias de infantería en la compañía del teniente coronel Armendáriz, a quien escribió mi hermano [Juan Bautista Villarreal], le cuida y le atiende. $\underline{35}$

En Cataluña entregó a su primer teniente Francisco Mertens dos cartas que llevaba para él. Una de ellas era de Armendáriz, "escrita de su puño y letra” en la que lo recomendaba con términos muy favorables, y la otra era de Gabriel de Zuloaga. Asimismo, fue a visitar a Andrés de Orbe y Larreategui, obispo de Barcelona y, como sabemos, muy amigo y favorecedor de la familia y a Cristóbal Lorenzo del Corral e Idiáquez (nacido en Villarreal de Urrechu, 1668), regente de la Audiencia de dicha ciudad $\underline{36}$.

Por otra parte, los ascensos y nombramientos de Armendáriz en diversos gobiernos políticos militares influyeron directamente en sus protegidos, por lo que los Villarreal de Bérriz y el resto de la parentela siguieron sus pasos detenidamente. Por este motivo, su nombramiento como capitán general de Guipúzcoa pudo ser muy beneficioso para los intereses de la familia y las otras casas amigas. De hecho, para asentar aún más los vínculos, Pedro Bernardo se planteó, en un principio, pasar a visitarlo a San Sebastián. Y lo mismo sucedió cuando en 1723, este navarro fue designado virrey del Perú, por razón de los importantes negocios que manejaban su casa y parentela en esas tierras $\frac{37}{3}$. Por una parte, esta protección se uniría los apoyos del primo y amigo Bruno Mauricio de Zavala, desde 1717 gobernador de Buenos Aires, así como otros vínculos con otras altas autoridades limeñas como, por ejemplo, el arzobispo Soloaga. Asimismo, en este camino de múltiples mediaciones, en las que se utilizaban distintos caminos para ayudar a parientes y amigos, el vínculo entre Pedro Bernardo y el nuevo virrey también será utilizado a favor de Bruno Mauricio y así se lo hace ver cuando le comunica que con el nuevo virrey, José de Armendáriz, “se holgará mucho”. $\underline{38}$ Desde entonces, la correspondencia entre la torre de Uriarte y la parentela del Perú se estableció a través de la casa del marqués de Castelfuerte y de su secretario José de Múxica.

Este conglomerado de vínculos que sustentaban dichas alianzas y asociaciones se fueron consolidando a lo largo de los años de diversos modos. Durante el desempeño de su virreinato, Armendáriz tuvo por paje a Pedro José Vega Romani, primo limeño de los marqueses de Feria y 
pariente de Villarreal de Bérriz, que además fue uno de sus acompañantes en su venida desde el Perú a España en 1736. En otro sentido, Miguel Gomendio Urrutia (Lima, 1670), alcalde del crimen de la Audiencia de Lima y pariente de Pedro Bernardo, fue el encargado de realizar la residencia del virrey Castelfuerte tras evitar a los oidores desafectos (Moreno; sala, 2004).

En los últimos meses de 1727, Peru tuvo orden de trasladarse a Madrid. De nuevo en la corte volvió a estar rodeado de los grandes amigos de la familia. Esta vez estuvo acogido en la casa de los Corral, primos de los Idiáquez, quienes le recibieron bien gustosos. Para entonces, estaba a punto de ajustarse la boda de su hermano Ignacio con Josefa de Corral Zarauz, a la vez que, José Joaquín, el único hermano de ésta, había pasado a Madrid para casarse con Teresa Rosalía de Aguirre, nieta de María Antonia de Salcedo, marquesa de Montehermoso $\underline{39}$. Sin embargo, ya habían pasado varios años y Peru ya se había ganado cierto respeto entre la parentela y así participaba como uno más en las reuniones y las decisiones que allí se tomaban.

La estancia de Peru en la casa de los Corral e Idiáquez fue aprovechada por los Villlarreal de Bérriz en la búsqueda de apoyos para sacar a Bruno Mauricio de Zavala de la gobernación del Río de la Plata, cansado ya de sus arduas labores en territorios de frontera, marcadas por las campañas de guerra y la lucha contra el contrabando.

De este modo, Pedro Bernardo comunicaba a su primo y amigo Bruno Mauricio:

"Escribiré a Peru, que en las conversaciones de Idiáquez y Areizaga hable de este punto a ver si se puede discurrir algún medio para traerle a v.m. a algún empleo correspondiente y también con el señor presidente Orbe, a quien debemos muchísimo y con grande cariño a instado a Peru sobre ponerle cama en si propio cuarto y aunque su empleo no tiene que ver con soldados, no dudo que si puede algo hará por v.m.” $(. . .)^{40}$.

Además de Idiáquez, está también su pariente y hombre de confianza Areizaga, quien había sido colocado por aquél en cargos de la casa del infante Don Fernando, futuro Príncipe de Asturias. Pedro Bernardo contó también con otro importante apoyo en la corte: el vizcaíno Andrés de Orbe y Larreategui, presidente del Consejo de Castilla, arzobispo de Valencia y también amigo de la familia que, además de su cargo, gozaba con buenos apoyos en puestos de poder. $\underline{41}$ Sin embargo, queda claro que en este caso en particular, esa no es la tecla que primero hay que tocar, ya que este asunto "es cosa de soldados', como dice la fuente.

La estrategia parece finalmente haber dado resultado, ya que al momento de su muerte en 1736, Bruno Mauricio de Zabala había sido nombrado presidente de la Capitanía General de Chile, un lugar de mayor jerarquía y la antesala probable de su vuelta a casa. Sin embargo, un factor no previsto rompió la cadena y la muerte fue el destino no deseado ni buscado.

\section{Notas}

1 Carta entre Bruno Mauricio de Zavala y Martín Aurelio de Maguna y Zavala 14 de marzo de 1723. Archivo de la Familia Ampuero. Sección Zabala. Fundación Sancho el Sabio. Vitoria-Gasteiz. 
$\underline{2}$ Desde hace años mi investigación se ha centrado principalmente en la participación de las elites vascas y navarras en la Monarquía Hispánica y sus consecuencias en las comunidades de origen (1650-1750) . Esta aportación tiene que ver con este devenir investigador. Entre otros, véanse Guerrero Elecalde, R. (2016), pp. 387-402; Guerrero Elecalde, 2009 (pp. 247-258), 2010a (pp. 145176), 2010b (pp. 125-145), 2012a y 2012b; Imízcoz; Guerrero, 2004, pp. 177-238.

3 Sobre la gobernación de Buenos Aires y el virreinato del Perú: Tarragó, G. (2017b); Tarragó, G. (2010), pp. 177-209.

4 Expediente de información y licencia de pasajero a indias del brigadier Bruno Mauricio de Zabala, gobernador y capitán general de la ciudad y puerto de Buenos Aires. Archivo General de Indias, Contratación, 5469,N.2,R.31. 7 de febrero de 1727.

5 Carta de Bruno Mauricio de Zavala y Martín Aurelio de Maguna y Zavala. 17 de febrero de 1717. Archivo de la Familia Ampuero, Sección Zabala. Fundación Sancho el Sabio. Vitoria-Gasteiz.

6 AHN, Órdenes Militares, Santiago, Exp. 4058, año 1708

Z Carta de Pedro de Idiáquez a Pedro Bernardo de Villarreal de Bérriz, Azcoitia, 2 de enero de 1704. Archivo de la Torre de Uriarte (ATU), leg. XXIV, 3-8-c, d.

$\underline{8}$ Archivo General de Simancas (AGS), Guerra Moderna, leg. 2.246, 13 de febrero de 1716.

9 Archivo Histórico Nacional, Madrid (AHN), Estado, Carlos III, exp. 510, año 1791.

10 Base de datos Fichoz.

11 AHN, Órdenes Militares, Calatrava, exp. 1.272, año 1701.

12 Durante la Guerra de Sucesión participó en los conflictos en el reino de Valencia, especialmente la acción de San Mateo y la toma de Villarreal en el Maestrazgo. Pasó luego a Madrid, donde fue hecho prisionero y luego rescatado. En 1707, participó del sitio de Lérida, acción donde perdió un brazo. Tarragó, G. (2017b).

$\underline{13}$ Fue elevado a teniente general en 1736.

14 Archivo General de Palacio (AGP), Caja 947, Exp.28.

15 AGP, Caja 947, Exp.28.

16 Archivo de la Casa de Alcibar-Jaúregui, Fondo de la Casa de Alcibar-Jaúregui, Carta de José Aguirre y Acharán a su hermano Pedro. Madrid, 27 de abril de 1728.

17 AHN, Órdenes Militares, Santiago, Exp. 4058, año 1708.

18 Base de Datos Fichoz.

19 Anteriormente, el 2 de mayo de 1648, María había casado en primeras nupcias con el capitán Mateo de Aranguren (Mondragón, 1612-1664), perteneciente a una familia amiga de la comarca, que desarrolló sus negocios en las Indias. De este enlace nacieron Ana María de Aranguren y Andicano, que contrajo matrimonio con José Manrique de Arana, marqués de Villalegre, teniente de la guardia española, paje de Felipe IV y embajador con Carlos II y Mateo Nicolás de Aranguren y Andicano (Mondragón, 1652-1736), que con apenas catorce años obtuvo el hábito de caballero de 
Santiago y el empleo de caballerizo del rey, que mantuvo a lo largo del reinado de Felipe V. AHN, Órdenes Militares, Santiago, exp. 523, año 1665.

$\underline{20}$ Base de datos Fichoz.

21 María Sáez de Andicano casó el 13 de enero de 1666 en segundas nupcias con Pedro de Villarreal de Bérriz Gamboa. AHN, Órdenes Militares, Santiago, exp. 8.940, año 1690.

$\underline{22}$ AHN, Órdenes Militares, Santiago, exp. 8.941, año 1681.

$\underline{3}$ De hecho, ya estuvieron establecidos como comerciantes en Manila, reino de Filipinas, Juan de Arranotegui y Bengolea y Juan de Bengolea. Archivo General de Indias (AGI), Contratación, 925, N.7 (Año de 1594).

24 Carta de Pedro Bernardo Villarreal de Bérriz a Francisco de Aguirre, Lequeitio, 1 de agosto de 1715. ATU, Copiadores de cartas escritas a Indias a diferentes personas y parientes por el señor D. Pedro Bernardo Villarreal de Bérriz, caballero del orden de Santiago y Dña. María Teresa de Bengolea y Esquível.

$\underline{25}$ Un ejemplo de este tipo de actuaciones y en otros territorios: Tarragó, G. 2017b).

26 Carta de Nicolás de Ubilla a Pedro Bernardo de Villarreal de Bérriz, Marquina, 19 de enero de 1703. ATU, leg. XXI, 1-30-a, b.

$\underline{27}$ Un antepasado fue Catalina de Arteaga Leiva y Gamboa, señora de Arteaga (en Vizcaya). Por derecho, esta familia poseía los prebostazgos de Guernica, Ondárroa y Bermeo.

28 Carta de Ignacio de Villarreal de Bérriz a su padre, Pedro Bernardo Villarreal de Bérriz, Madrid, 17 de marzo de 1721. ATU, leg. XXIII, 6-26-a, b, c, d, e, f y g.

29 Carta de Ignacio de Villarreal de Bérriz a su padre, Pedro Bernardo Villarreal de Bérriz, Madrid, 16 de diciembre de 1720. ATU, leg. XXIII, 6-22-a, b, c, d, e, f, g, y h.

30 Carta de Ignacio de Villarreal de Bérriz a su padre, Pedro Bernardo Villarreal de Bérriz, Madrid, 8 de diciembre de 1721. ATU, leg. XXIII, 6-37-a, b, c y d.

31 Carta de Ignacio de Villarreal de Bérriz a su padre, Pedro Bernardo Villarreal de Bérriz, Madrid, 17 de marzo de 1721. ATU, leg. XXIII, 6-26-a, b, c, d, e, f y g.

$\underline{32}$ Carta de Ignacio de Villarreal de Bérriz a su padre, Pedro Bernardo Villarreal de Bérriz, Madrid, 31 de marzo de 1721. ATU, leg. XXIII, 6-28-a, b, c, d, e, f y g.

33 Carta de Ignacio de Villarreal de Bérriz a su padre, Pedro Bernardo Villarreal de Bérriz, Madrid, 18 de agosto de 1721. ATU, leg. XXIII, 6-34-a, b, c y d.

34 Carta de Ignacio de Villarreal de Bérriz a su padre, Pedro Bernardo Villarreal de Bérriz, Madrid, 31 de marzo de 1721. ATU, leg. XXIII, 6-28-a, b, c, d, e, f y g.

35 Carta de Pedro Bernardo Villarreal de Bérriz a Bruno Mauricio de Zavala, Lequeitio, 3 de abril de 1721. Archivo de la Torre de Uriarte, Lequeitio, Copiador de cartas con los parientes de Indias. 
36 Carta de Pedro José Villarreal y Bengolea a su padre Pedro Bernardo Villarreal de Bérriz, Barcelona, 4 de octubre de 1721. Citado en Ruiz de Azúa, E. (1990), p. 94.

37 Carta de Pedro Bernardo de Villarreal de Bérriz a Santiago de Bengoa, 7 de octubre de 1723. ATU, Copiador de cartas con los parientes de Indias.

38 Carta de Pedro Bernardo de Villarreal de Bérriz a Bruno Mauricio de Zavala, 9 de diciembre de 1723. Archivo de la Torre de Uriarte, Lequeitio, Copiador de cartas con los parientes de Indias.

39 Carta de Pedro Bernardo Villarreal de Bérriz a Bruno Mauricio de Zavala, Lequeitio, 18 de marzo de 1728. ATU, Copiador de cartas con los parientes de Indias.

40 Carta de Pedro Bernardo Villarreal de Bérriz a Bruno Mauricio de Zavala, Lequeitio, 18 de marzo de 1728. ATU, Copiador de cartas con los parientes de Indias.

$\underline{41}$ Entre otros, el guipuzcoano Juan Bautista de Orendain, secretario del Despacho de Estado.

\section{Bibliografía}

Albareda, J. (2010): La Guerra de Sucesión de España (1700-1714), Madrid, Ed. Crítica.

Andújar, F. (2009): “De la periferia a la Corte: la integración de vascos y navarros en los cuerpos de elite del ejército borbónico”, en: Porres, R. y Reguera, I. (eds.), La proyección de la Monarquía Hispánica en Europa. Política, Guerra y Diplomacia entre los siglos XVI y XVIII, Bilbao, Ed. Universidad del País Vasco. pp. 175-196.

Andújar, F. (2000): “Élites de poder militar: las guardias reales en el siglo XVIII”, en Castellano, J.L.; Dedieu, J.P.; López-Cordón, M.V. (eds.), La pluma, la mitra y la espada. Estudios de Historia Institucional de la Edad Moderna, Madrid, Marcial Pons, pp. 65-94.

Andújar, F. (2004): El sonido del dinero. Monarquía, ejército y venalidad en la España del siglo XVIII, Madrid, Marcial Pons.

Arrieta, J. (2009-2010): “Entre monarquía compuesta y estado de las autonomías. Rasgos básicos de la experiencia histórica española en la formación de una estructura política plural”, en IVS FVGIT Revista de Estudios Histórico-Jurídicos de la Corona de Aragón, vol. 16, pp. 9-72

Arrieta, J., Gil, X., Morales, J. (coords.) (2017): La diadema del rey. Vizcaya, Navarra, Aragón y Cerdeña en la Monarquía de España (siglos XVI-XVII), Bilbao, Ed. Universidad del País Vasco.

Badorrey Martín, Beatriz (1999): Los orígenes del Ministerio de Asuntos Exteriores (1714-1808), Madrid, Ministerio de Asuntos Exteriores.

Barriera, D. (2002.): Vers une histoire politique configurationnel. Conquérants, familles et rapports de pouvoir dans une ville aux confins de ÍEmpire Espagnol - (Santa Fe, Río de la Plata, XVI-XVII siècles), Thèse de Doctorat, realizada bajo la dirección de Bernard Vincent y María Inés Carzolio, Paris, EHESS. 
Barriera, D. (2002b): "Por el camino de la historia política: hacia una historia política configuracional”, en: Secuencia, nueva época, núm. 53, pp. 163-196.

Benigno, F. (1994): La sombra del rey, Madrid, Alianza Editorial.

Castro, C. (2004): A la sombra de Felipe V. José de Grimaldo, ministro responsable (1703-1726), Madrid, Marcial Pons.

Clavero, B. (1991): Antidora, Antropología Católica de la Economía Moderna, Milán, Giuffre Editore.

Dedieu, J.P. (2000): "La Nueva Planta en su contexto. Las reformas del aparato del Estado en el reinado de Felipe V”, en: Manuscrits: Revista d'història moderna, núm. 18, pp. 113-139.

Desos, C. (2009): Les Français de Philippe V. Un modèle nouveau pour gouverner l'Espagne (1700-1724), Estrasburgo, Presses Universitaires de Strasbourg.

Dubet, A. (2005): “Administrar los gastos de guerra: Juan Orry y las primeras reformas de Felipe V (1703-1705)”, en: Actas de la VIIIa Reunión Científica Fundación Española de Historia Moderna. Madrid, 2-4 junio 2004, vol. II, FEHM, Madrid.

Dubet, A. (2008): Un estadista francés en la España de los Borbones. Juan Orry y las primeras reformas de Felipe V (1701-1706), Madrid, Biblioteca Nueva.

Esteban, Alicia y Ruiz Ibáñez, José Javier (1998): “El gobierno político y militar de los Países Bajos. La gestión Administrativa e institucional de un territorio periférico de la Monarquía Católica (siglos XVI-XVII), en: Relaciones. Estudios de Historia y Sociedad, vol. XIX, núm. 73, invierno, pp. 115-167.

Esteban, A. (coord.) (2012): Servir al rey en la Monarquía de los Austrias: Medios, fines y logros del servicio al soberano en los siglos XVI y XVII, Madrid, Ed.Sílex.

Fernández Albaladejo, P. (ed.) (2002): Los Borbones. Dinastía y memoria de la nación en la España del Siglo XVIII, Madrid, Marcial Pons Historia/Casa Velázquez.

García Fuentes, L. (1991): Sevilla, los vascos y América. (Las exportaciones de hierro y manufacturas metálicas en los siglos XVI, XVII y XVIII), Bilbao, Fundación BBV.

Gil, X. (2013): “Integrar un mundo. Dinámicas de agregación y de cohesión en la Monarquía de España”, en: Mazín, O. y Ruiz Ibáñez, J.J. (eds.): Las Indias Occidentales. Procesos de incorporación territorial a las Monarquías Ibéricas, México, El Colegio de México/Red Columnaria, pp-69-108.

Gil. X. (2017): “De diademas compuestas y circunferencias, de provincias y periferias”, en: Arrieta, J., Gil, X., Morales, J. (coords.) (2017): La diadema del rey. Vizcaya, Navarra, Aragón y Cerdeña en la Monarquía de España (siglos XVI-XVII), Bilbao, Ed. Universidad del País Vasco, pp.17-52.

Guerrero Elecalde, R.(2010): “Los hombres del rey. Redes, poder y surgimiento de nuevas elites gobernantes durante la guerra de sucesión española (1700-1714)”, en: Prohistoria. Historia-Política de la Historia, Año XIII, num. 13, pp. 125-145 
Guerrero Elecalde, R. (2012): Las elites vascas y navarras en el gobierno de la Monarquía borbónica: Redes sociales, carreras y hegemonía en el siglo XVIII (1700-1746). Bilbao, Ed. Universidad del País Vasco.

Guerrero Elecalde, R; Tarragó G. (2014): “La parte del león: vizcaínos en la disputa por el comercio con Buenos Aires (1720-1750)”, en: Revista Història, Històrias, vol. 2, no. 3.

Guerrero Elecalde, R. (2017): "Familias en la corte y gobierno provincial. Patronazgo, influencia y articulación de los territorios (Las provincias vascas, 1700-1746)”, en: Favarò, V.; Merluzzi, M.; Sabatini, G. (eds.): Fronteras. Procesos y prácticas de integración y conflictos entre Europa y América (Siglos XVI-XX), Madrid, FCE/ Red Columnaria, pp. 387-402.

Hespanha, A.M. (1993): La gracia del derecho: economía de la cultura en la edad moderna, Madrid, Centro de Estudios Constitucionales.

Imízcoz, J.M.; Guerrero Elecalde, R. (2004): “Familias en la Monarquía. La política familiar de las elites vascas y navarras en el Imperio de los Borbones”, en: Imízcoz, José María (dir.): Casa, Familia y Sociedad (País Vasco, España y América, siglos XV-XIX), Bilbao, Ed. Universidad del País Vasco, pp. 177- 238.

Imízcoz, J.M. (2001), "Patronos y mediadores: redes familiares en la Monarquía y patronazgo en la aldea: la hegemonía de las elites baztanesas en el siglo XVIII», en IImízcoz, J.M. (coord.), Redes familiares y patronazgo: aproximación al entramado social del País Vasco y Navarra en el Antiguo Régimen (siglos XV-XIX), Bilbao, Universidad del País Vasco Ed., pp. 225-262.

Imízcoz, J.M. (2011): “Actores y redes sociales en Historia”, en: Carvajal, D.; Añibarro , J.; Vítores, I. (eds): Redes sociales y políticas en el mundo bajomedieval, Castilla Ediciones, Valladolid, pp.2033

Imízcoz, José María (2008): “Las élites vasco-navarras y la monarquía hispánica: construcciones sociales, políticas y culturales en la edad moderna”, en: Cuadernos de Historia Moderna, vol. 33, pp. 89-119.

Kamen, H. (1974): La guerra de Sucesión en España 1700-1715, Barcelona, Grijalbo.

Kamen, H. (2000): Felipe V: el rey que reinó dos veces, Madrid, Temas de Hoy.

Larrañaga, K. (1974): “Dos caballeros vascos en el mundo del barroco, los hermanos Juan Bautista y Pedro Bernardo Villarreal”, BRSBAP, Año 30, cuaderno 3-4 , p. 291-335.

López Cordón, M.V., "Secretarios y Secretarías en la Edad Moderna: de las manos del Príncipe a los relojeros de la Monarquía”, en: Studia Historica, Historia Moderna, num.15, 1996, pp. 107-133.

Martínez Shaw, C.; Alonso Mola, M. (2001): Felipe V, Madrid, Arlanza.

Mazín Gómez, Óscar (1998): “Introducción”, en: La monarquía española: grupos locales ante la corte de Madrid, Relaciones-Estudios de Historia y Sociedad, Relaciones, vol. XIX, núm. 73, El Colegio de Michoacán, pp. 11-14.

Moreno, A.; Sala, N. (2004): El «premio» de ser virrey: los intereses públicos y privados del gobierno virreinal en el Perú de Felipe V, Madrid, Instituto de Historia. 
Moutoukias, Z. (2002): "Las formas complejas de la acción política: justicia cor-porativa, faccionalismo y redes sociales (Buenos Aires, 1750-1760)”,en: Jahrbuch Für Geschichte Lateinsamerikas, núm. 39, pp. 69-102.

Ruiz de Azúa, E. (1990) D. Pedro Bernardo Villarreal de Bérriz (1669-1740). Semblanzas de un Vasco Precursor, Madrid, Editorial Castalia/Fundación Juanelo Turriano.

Tarragó, G. (2010): "Redes mercantiles y prácticas empresariales: comerciantes rioplatenses del siglo XVIII”, en: Actas del Seminario Internacional "Compredere le Monarchie Iberiche. II Sesione. Risorse Materiali e reppresentazione del potere”, Milán, Universitá Roma III/Red Columnaria, Viella, pp. 309-340.

Tarragó, G.(2012): “Espacio, recursos y territorio: la Gobernación del Río de la Plata durante el reinado de Felipe V”, en: Mazín, O. y Ruiz Ibáñez, J.J.: Las Indias Occidentales. Procesos de incorporación territorial a las Monarquías Ibéricas, México, El Colegio de México/Red Columnaria, pp. 281-327

Tarragó, G. (2017a): "Hierro vizcaíno/plata potosina: ferrones y empresarios vascos en la reconfiguración de un territorio americano (Río de la Plata, virreinato del Perú, 1700-1745), en: Favarò, V.; Merluzzi, M.; Sabatini, G. (eds.) (2017): Fronteras. Procesos y prácticas de integración y conflictos entre Europa y América (Siglos XVI-XX), Madrid, FCE/ Red Columnaria, pp. 519532.

Tarragó, G. (2017b): La gobernación del Río de la Plata durante el reinado de Felipe V (17001746): tramas vinculares, configuraciones políticas y análisis microsocial. Tesis doctoral en curso bajo la dirección de José María Imízcoz, Departamento de Historia, Medieval, Moderna y de América, Facultad de Letras, Universidad del País Vasco.

Yun, B. (2009): “Introducción”, en: Entre el imperio colonial y la monarquía compuesta. Élites y territorios en la Monarquía Hispánica (ss. XVI-y XVII), en: Yun Casalilla, Bartolomé, Las redes del imperio. Élites sociales en la articulación de la Monarquía Hispánica, 1492-1714, Marcial PonsUniversidad Pablo Olavide. 